\title{
Sevend Pounds y Biología. Zoología y transplante de órganos en el epílogo del film, una experiencia en formación de docente
}

Seven Pounds and Biology. Zoology and organs transplant at the epilogue of this film, an experience at a teacher training course

Javier Grilli Silva

Ce.R.P del Litoral - Salto, Uruguay

Resumen: Las experiencias didácticas vividas en formación docente inicial tienen un fuerte impacto en la conformación del perfil profesional del profesor. Estas deben ser coincidente con los fines que se tienen para la enseñanza de las ciencias en el nivel educativo medio: formar ciudadanos críticos. Propuestas de enseñanza de la zoología con un enfoque CTS ofrecen una visión integrada, interdisciplinar, y problematizadora del saber científico, que conectan la ciencia con la realidad que vive el individuo en la sociedad. La utilización del cine como recurso didáctico permite vincular de manera muy favorable los avances científicos y tecnológicos con aspectos sociales. La experiencia didáctica que presentamos desarrolló una mirada crítica al film Seven Pounds con soporte en la Zoología, Medicina y el Derecho. Por medio de una guía se realizó el visionado del film, lográndose movilizar conocimientos científicos, reflexionar con base en ellos y aplicarlos o usarlos en situaciones específicas.

Palabras clave: Zoología, CTS, cine, recurso didáctico, espectador crítico.

Abstract: Didactic experiences at teaching training courses have a great impact on the profile building up of a future teacher. Those experiences must coincide with the means which are available to teach science at a secondary level education: to develop critical citizens. Educational proposals in zoology with a STS approach provide an integrated vision, interdisciplinary and scientific knowledge problematizing, which made a connection between science and reality in which people are immerse in society. The use of films as a didactic source, allows connecting in a very positive way scientific and technological progress with social issues. This didactic experience, which we have showed has developed a critical gaze to the film Seven Pounds, with support in Zoology, Medicine and Law. The film was seen with the help of a guide, making scientific knowledge mobilize, and reflect on them to later on apply them on specific situations.

Keywords: Zoology, STS, films, didactic source, critical audience.

(Fecha de recepción: julio, 2014, y de aceptación: noviembre, 2014)

DOI: 10.7203/DCES.29.3911 


\section{Introducción}

En Uruguay la Formación Docente pública está regulada por un plan de estudio, el Plan 2008, de carácter nacional $^{1}$ y con una duración total para la carrera de 4 años. El Plan comprende la formación de maestros (para el nivel educativo inicial y primario), profesores (para el nivel educativo medio) y maestros técnicos (para la educación técnica, media y superior).

La formación de un Profesor de Biología incluye asignaturas de ciencias de la educación (Sociología, Pedagogía, etc.), instrumentales para la docencia (Informática, Inglés), la unidad conformado por didáctica - práctica docente, y asignaturas específicas. Con relación a estas últimas, el Plan presenta una clara fragmentación de los saberes dentro de la Especialidad. Por este motivo y poniendo un ejemplo, el estudio de la biología animal está comprendido en al menos tres asignaturas y en dos años diferentes: Zoología I (Zoo-I de aquí en delante), Zoología II y Microbiología.

En Zoo-I se estudian animales protostomados. Es una asignatura estructurada de manera clásica, vertical: considera la anatomía y fisiología de los Phylum, desde aquellos de niveles de organización más sencillos hacia aquellos más complejos. En la fundamentación del Programa se dice:

El curso entonces será desarrollado con especial énfasis en la biología de cada Phylum, incluyendo en ésta el estudio de las estructuras anatómicas, modificaciones fisiológicas, ciclos vitales y aspectos vinculados al comportamiento, que le permiten su éxito evolutivo. Programa Oficial de la Asignatura Zoología I - Plan $2008^{2}$

Como en otros cursos básicos de zoología de nivel terciario ${ }^{3}$, en Zoo-I se busca el aprendizaje de conocimientos que permiten interpretar el plan estructural y funcional de los distintos Phylum. Se plantea analizar comparativamente los rasgos morfológicos y anatómicos de los diferentes taxa así como su significado adaptativo que les permite a los organismos cumplir con los requerimientos fisiológicos básicos.

Cursos de zoología en la formación de agrónomos y veterinarios, presentan un enfoque y selección de contenidos que transforman la asignatura en una zoología aplicada. Por su parte en formación docente algunas propuestas

\footnotetext{
${ }^{1}$ Se aplica en todos los Centros de Formación Docente del país: IPA (Instituto de Profesores Artigas en Montevideo), IFDs (Institutos de Formación Docente, en los Departamentos del Interior), CERPs (Centros Regionales de Profesores, con cobertura regional en el Interior), INET (Instituto Normal de Enseñanza Técnica en Montevideo) e Institutos Normales de Montevideo.

${ }^{2}$ Disponible en http://www.cfe.edu.uy/images/stories/pdfs/planes_programas/profesorado/plan_2008/biologia/tercero/ zoologia_I.pdf

${ }^{3}$ Ver por ejemplo "Zoología Invertebrados II", Facultad de Ciencias Naturales y Museo de la Universidad Nacional de La Plata (en http://www.fcnym.unlp.edu.ar/uploads/docs/programa_artropodos_2000.pdf); o "Zoología" Licenciatura de Biología en la Universidad del país Vasco (en http://www.irakasleak.ehu.es/p253-content/es/contenidos/ informacion/00076_docencia/es_00076_do/00076_docencia.html)
} 
proponen una zoología que sirva de sustento a una mejor comprensión de temas ecológicos (Lugo, Hernández y Álvarez, 2012). En el presente trabajo presentamos una propuesta que plantea un enfoque alternativo para el abordaje de algunos temas de zoología.

\section{Para qué enseñar zoología}

En los estudiantes del nivel educativo medio de Uruguay se formula una pregunta clásica a los profesores, acompañada de su respuesta que también es clásica: “¿Para qué sirve esto, profesor?.... Ya lo verás más adelante”. El entrecomillado es además el subtítulo que puso Acevedo Díaz (2004) a uno de sus trabajos donde trata los fines de la enseñanza de la ciencia en el nivel educativo medio. La respuesta dada por el profesor a la pregunta formulada por el adolescente, es un fiel reflejo de los fines que en las aulas tiene la enseñanza de las ciencias: propedéutico, preparatorio para la universidad. Será entonces luego, en el nivel educativo superior, donde el joven verá la aplicación del conocimiento que se le enseña.

La pregunta "¿para qué sirve esto profesor?" transmite una preocupación sincera y legítima del alumno. Podemos re-escribir e interpretar su pregunta en otras dos: ¿qué utilidad tiene esto que el profesor enseña?, y ¿qué vínculo o conexión tiene esto con lo que a mí me interesa saber?

Como cambio al enfoque tradicional propedéutico que se da en la enseñanza de las ciencias correspondiente a los niveles educativos básicos, Acevedo Díaz plantea una ciencia escolar para la ciudadanía: interesante a los estudiantes, aplicativa a situaciones reales, útil para resolver situaciones, para tomar decisiones. Una ciencia que forme al joven para adherir o rechazar posturas existentes en la sociedad, con base en pruebas y argumentos racionales. Podemos sintetizar la idea diciendo que se trata de aprender ciencia para entender y desenvolverse convenientemente en la realidad que se está inmerso.

El enfoque de enseñanza de las ciencias que venimos considerando, se entronca con otros dos ampliamente tratados en la literatura: la alfabetización científica y la enseñanza CTS (Ciencia, Tecnología y Sociedad).

La epistemología de los últimos decenios ha dejado muy en claro la necesidad de concebir la ciencia y la actividad científica en general como construcciones humanas y no como descubrimientos de leyes pre-existentes. En consecuencia con esto la actividad científica no es imparcial o neutra y se enmarca en los momentos socio-políticos-culturales particulares en las que transcurre; el condicionamiento e influencia de la sociedad está de una forma u otra siempre presente.

El desarrollo científico y tecnológico no puede ser concebido sin considerar a la sociedad, ya sea esta como receptor de conocimiento, como usuario, o como promotora facilitadora del mismo. La búsqueda del conocimiento y la creación están ligados, cada vez más, a las necesidades sociales. 
Esta postura epistemológica se ve muy bien incorporada en el movimiento académico y educativo de fines de los 60 y principios de los 70: CTS (López Cerezo, 1999). El mismo se constituye en un campo de trabajo, donde se trata de entender el fenómeno científico-tecnológico en su contexto social, tanto en relación con sus condicionantes sociales como en lo que atañe a sus consecuencias sociales y ambientales.

En el enfoque CTS es posible identificar dos grandes tradiciones que representan dos formas distintas de entender la contextualización social de la cienciatecnología. Una de las tradiciones es de origen europeo y la otra norteamericana (González, López y Luján, 1996). Desde una perspectiva educativa las dos miradas constituyen alternativas complementarias. Ambas promueven la conexión entre la ciencia y la realidad que vive el individuo en la sociedad.

\section{Zoología desde una perspectiva CTS}

La enseñanza de la zoología (así como otras disciplinas), debe facilitar y promover claramente la conexión entre ciencia y la realidad que vive el alumno en la sociedad a la que pertenece. La zoología debe contribuir a que los alumnos tengan una mirada de cuestiones cotidianas con un soporte o sustento desde la construcción humana que llamamos ciencia.

Desde 2011 a 2014 en el curso de Zoo-I venimos abordando algunos temas programáticos desde una mirada
CTS. A principio del año lectivo se conforman equipos de trabajo que realizan una investigación bibliográfica y recolección de datos locales, para uno de 6 tópicos (o temas) que se sortean entre los estudiantes. (Cuadro I)

Estos 6 tópicos CTS se incorporan estratégicamente en el desarrollo de las unidades temáticas planteada por el Programa Oficial de la Asignatura Zoo-I. Como ya mencionamos, Zoo-I tiene una secuencia de temas con base en el nivel de organización corporal de los animales protostomados. Se estudian los grandes Phylum comenzando con aquellos de organización corporal celular y tisular (Porífera y Cnidaria), luego los de organización orgánica (Platyhelminthes) y en el último tramo del año se abordan los de organización sistémico-orgánico (Nemátoda, Annelida, Mollusca y Artrópoda). Cada uno de los 6 tópicos CTS que ideamos se trabaja en clase con la totalidad del grupo - y teniendo especial participación del equipo que realizó la investigación- durante el abordaje del Phylum con el que tiene correspondencia. La propuesta concluye con la entrega por parte de los equipo de un trabajo monográfico a fin de año.

Uno de los tópicos, el $n^{\circ} 5$, considera la aplicación biotecnológica en un tema de interés particular para la región noroeste del país: la utilización de insectos como controladores biológicos de otros insectos que son plaga en la citricultura. Otro de los tópicos ( $\left.\mathrm{n}^{\circ} 6\right)$, apunta al conocimiento de las especies de arañas potencialmente peligrosas para la población, así como el conocimiento de las medidas 


\section{Cuadro I. Lista de temas CTS para el curso de Zoo-I - 2014}

1. Cine y zoología: Seven pounds y la "avispa de mar".
a) Ficha técnica de la película
b) Trama argumentativa del film; epílogo.
c) Bases biológicas del epílogo: toxicidad de la avispa de mar y diferentes aspectos vinculados con la donación de órganos a "corazón parado".
d) Ubicación taxonómica y geográfica de la avispa de mar.
e) Características anatómicas del grupo Cubozoa.

2. La religión judía y la ausencia de parasitosis por helmintos vinculados con el consumo de carne de cerdo.
a) Preceptos religiosos de la doctrina judía vinculados con el consumo de carnes.
b) Conocimiento científico vinculado a cuestiones epidemiológicas-sanitarias y preceptos religiosos que involucran estas cuestiones.
c) Aspectos epidemiológicos de triquiniasis y teniasis en la población hebrea y uruguaya.
d) Características de las larvas y del ciclo biológico de los Platelmintos Taenia solium y Trichinella spiralis.
e) Profilaxis (prevención primaria) para triquiniasis y teniasis por solium.

3. Lombricultura y vermicompost.
a) Conceptos generales sobre cría de lombrices.
b) La lombriz californiana y otras especies usadas en lombricultura; características anatómicas y fisiológicas que las hacen excelentes recicladoras de materia orgánica.
c) Vermicompost y agricultura orgánica.
d) Construcción de vermicompostera para reciclar residuos hogareños.

4. La comunicación visual en los cefalópodos.
a) Conceptos claves en la comunicación visual: emisor, receptor, mensaje, código y canal.
b) Funciones de la comunicación visual en cefalópodos.
c) El lenguaje visual en las sepias.
d) El rey de la imitación en los animales invertebrados: Thaumoctopus mimicus
e) Comunicación visual en humanos: aspectos biológicos comparables a cefalópodos

5. "Insectos insecticidas", una forma de control biológico de importancia para la citricultura salteña.
a) Características de la afectación del citrus por cochinilla roja californiana.
b) El uso de controles químicos para la cochinilla roja. Inconvenientes o prejuicios.
c) Concepto de parasitoidismo.
d) Características de la cochinilla roja con énfasis en aquellos momentos del ciclo pasibles de ser controlados por parasitoides.
e) Estrategia de control biológico de la cochinilla en Salto.

6. Arañas peligrosas del Uruguay.
a) Especies de arañas peligrosas en Uruguay: características diagnóstico y ubicación taxonómica.
b) Características de la ponzoña y del aracnidismo para cada una de las especies.
c) ¿Qué hacer y qué no hacer, como primeros auxilios, ante un caso de accidente por araña?
d) Aracnofobia y su consideración en contextos educativos. 
de prevención primaria y secundaria para el aracnidismo. Cuestiones de biotecnología como la producción de suero son incluidas en este tópico. Se incorporan estos dos tópicos en el curso cuando se trabaja el Phylum Artrópoda. En fin, otro tópico focaliza en el conocimiento de prácticas alimenticias de determinadas comunidades existentes en el país viendo el sustento que las mismas pueden tener en ciencias biomédicas (tópico n²); otro focaliza en la utilización de las lombrices de tierra para la generación de compost a partir de los residuos orgá- nicos domiciliarios (tópico $\mathrm{n}^{\circ} 3$ ) y otro en los aspectos biológicos de fondo que existen en la comunicación visual de cefalópodos y humanos (tópico $\mathrm{n}^{\circ} 4$ ). En todos los casos los conocimientos básicos de zoología, tanto en los aspectos de anatomía (macro y micro) como de fisiología, son el fundamento o sustento de las aplicaciones tecnológicas y/o de las prácticas sociales que se consideran en el tópico. El momento en el transcurso del año que se aborda cada tópico se corresponde con el momento en que el grupo animal (Phylum), es enseñado.

\section{Cuadro II. Ficha técnica y afiche del film Seven Pounds}

\begin{tabular}{|c|c|}
\hline TÍTULO ORIGINAL & Seven Pounds \\
\hline AÑo & 2008 \\
\hline DURACIÓN & $118 \mathrm{~min}$. \\
\hline PAÍS & 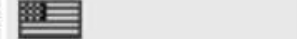 \\
\hline DIRECTOR & Gabriele Muccino \\
\hline GUIÓN & Grant Nieporte \\
\hline MÚSICA & Angelo Milli \\
\hline FOTOGRAFIA & Philippe Le Sourd \\
\hline REPARTO & $\begin{array}{l}\text { Will Smith, Rosario } \\
\text { Dawson, Wo } \\
\text { ody Harrelson, Barry } \\
\text { Pepper, Elpidia } \\
\text { Carrillo, Connor Cruise, } \\
\text { Michael Ealy, Robinne } \\
\text { Lee, Joe Nunez }\end{array}$ \\
\hline PRODUCTORA & $\begin{array}{l}\text { Columbia Pictures / Relativity } \\
\text { Media / Overbrook } \\
\text { Entertainment/ Escape Artists }\end{array}$ \\
\hline WEB OFICIAL & http://www.sevenpounds.com/ \\
\hline GÉNERO & $\begin{array}{l}\text { Drama. Romance | Drama } \\
\text { romántico. Enfermedad }\end{array}$ \\
\hline
\end{tabular}

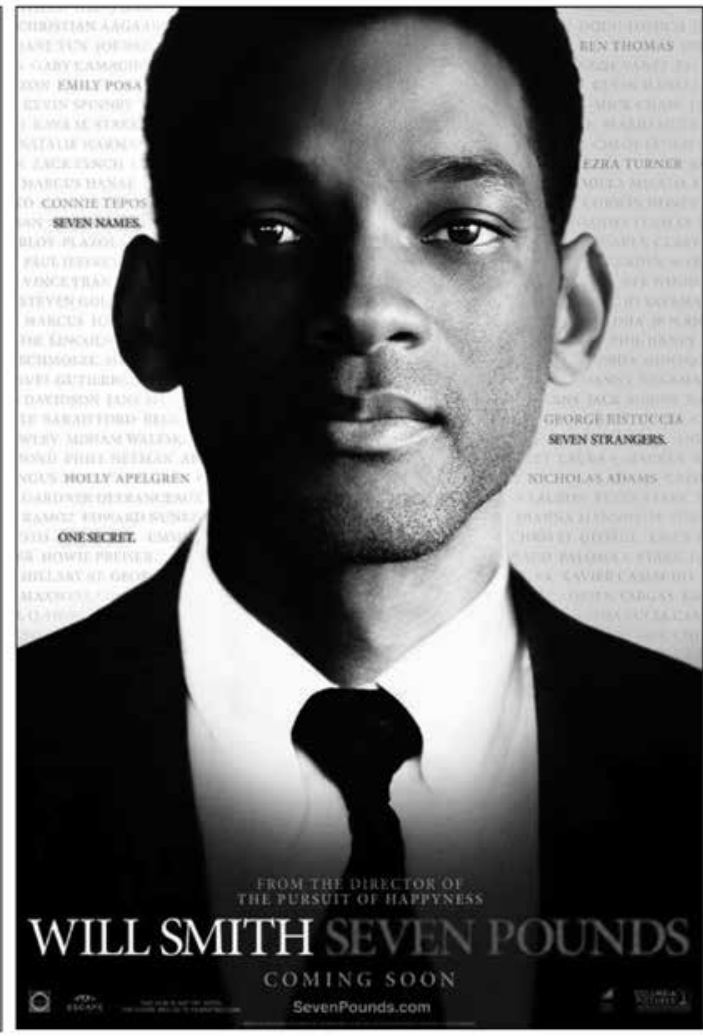

Fuente: Film affinity - http://www.filmaffinity.com/es/film171299.html 
Explicaremos la instrumentación dada al tópico $\mathrm{n}^{\circ} 1$. Al comenzar el estudio del grupo Cnidarios se propone a los estudiantes realizar en sus casas el visionado del film Seven Pounds (conocido en países de habla hispana como Siete almas). Cuadro II. Unas tres semanas después se realiza en clase con todo el grupo el visionado de un objeto de aprendizaje armado en movie-maker ${ }^{4}$. En él se incluye: el tráiler de la película, videos de entrevista a los dos protagonistas principales, fragmento de los últimos 12 minutos del film y textos con valoraciones de la película realizadas por críticos especializados en cine (Cuadro III).

Teniendo como referencia los ítems que se proponen para realizar la investigación bibliográfica (Cuadro I), se cuestiona la validez científica de lo que se propone en el epílogo de la película. Para esto se formulan preguntas guías

\section{Cuadro III. Opiniones sobre el film Seven Pounds de crítica especializada en cine}

I- "Algunas personas la encontrarán emocionalmente manipuladora. A algunas personas les gusta ser manipuladas emocionalmente. A mí sí, cuando está bien hecha. (...) Puntuación: *** (sobre 4).” (Roger Ebert: Chicago Sun-Times)

II- "Puede que esté entre las películas del tipo 'trascendente, formidable, llama a tu amigo a mitad de la noche para hablar sobre ella' más horribles jamás hechas. Te diría que fueras a verla para comprobarlo por ti mismo, pero puede que te lo tomes como una recomendación" (A. O. Scott: The New York Times)

III- "Es un pastel empapado de múltiples capas de sentimentalismo y coronado de una indigerible grandiosidad." (Joe Morgenstern: The Wall Street Journal)

IV- "Una fábula plenamente sentimental sobre el sacrificio y la redención que apunta al corazón a costa de la mente. Construida de forma tan intrincada que enfurecerá a cualquiera que se guíe por la racionalidad y el intelecto." (Todd McCarthy: Variety)

V- "La redención emprendida por el personaje principal nunca llega a ser moral, porque no hay trascendencia suficiente. Sólo es sentimental. (...) Todo conduce a una apoteosis de lo cursi que, al menos, es absolutamente sincera." (Javier Ocaña: Diario El País)

VI- "En la primera secuencia el filme ya anuncia sus macabras pero honradas intenciones (...) Muccino hace lo que puede con tal material sensible, aunque su mayor virtud es reclutar a la maravillosa Rosario Dawson (...) Puntuación: ** (sobre 5).” (Javier Cortijo: Diario ABC)

Fuente: http://www.filmaffinity.com/es/film171299.html

4 Entendemos por objeto de aprendizaje a "cualquier recurso digital que puede ser reusado como soporte para el aprendizaje" (Wiley, 2000). 


\section{Cuadro IV. Preguntas guías de análisis del film Seven Pounds}

1. ¿Qué tipo de Cnidario utiliza Tim para su propósito final?

2. ¿Qué características anatómicas diferencian estos cnidarios con las scifomedusas e hidromedusas ya estudiadas?

3. ¿Es posible la muerte de un humano por estos Cnidarios? ¿Qué características tiene el veneno producido por este tipo de animales?

4. ¿Qué tipo de trasplante se da en el epílogo?

5. Desde el punto de vista de la Medicina, ¿es posible una situación como la planteada en el epílogo del film?

6. ¿Qué establece la legislación internacional y nacional al respecto de los trasplantes en situaciones como la planteada en el film?

7. ¿Con cuál de las opiniones de críticos de cine que se presentó al final del audiovisual, acuerda más?

Fuente: elaboración propia

del visionado que orientan la mirada crítica del film. Cuadro IV.

Los estudiantes que realizaron la investigación bibliográfica desempeñan un papel preponderante guiando en clase la discusión, ofreciendo información -con su respectiva fuente- $\mathrm{y}$ ampliando el análisis a otras cuestiones de corte social, psicológica y artística. Se utilizan en el transcurso de la clase otros recursos complementarios para abordar los conceptos disciplinares de la zoología: dibujos esquemáticos $\mathrm{y}$ fotos de la especie animal en estudio. Cuadro V.

\section{Seven Pounds: una mirada desde la Zoología, Medicina y el Derecho}

El personaje principal de la película Seven Pounds es Ben Thomas (interpretado por Will Smith), un agente del IRS (Internal Revue Service) que se embarca en un viaje extraordinario de redención. El personaje interpretado por Smith en verdad es Tim Thomas, hermano de Ben, un ingeniero de la aeronáutica que por una imprudencia al volante provoca un catastrófico accidente de carretera en el que fallecen su propia esposa Sarah y otras seis personas más. Profundamente traumatizado por esta tragedia, suplanta la identidad de su hermano con el objetivo de obtener datos de siete personas que necesitan ayuda urgente, de tipo económico, médico o social. Mejorar estas siete vidas se convierte en su objetivo de vida. Para que todo transcurra de acuerdo con su voluntad, Tim cuenta con la ayuda de Dan (Barry Pepper), amigo de la infancia que deberá supervisar todo el proceso.

Ben selecciona a siete extraños a quienes investiga y entrevista con la aparente intención de ayudarlos; entre 


\section{Cuadro V. Fotografía de Chironex fleckeri.}

Soporte visual usado para enseñar características anatómicas macroscópicas de la especie. Umbrella de forma cúbica, tonalidad azul-verdoso y traslúcida; mechones de tentáculos de unos $80 \mathrm{~cm}$ de largo, son aspectos anatómicos de la especie que taxonómicamente se ubica en la Clase Cubozoa.

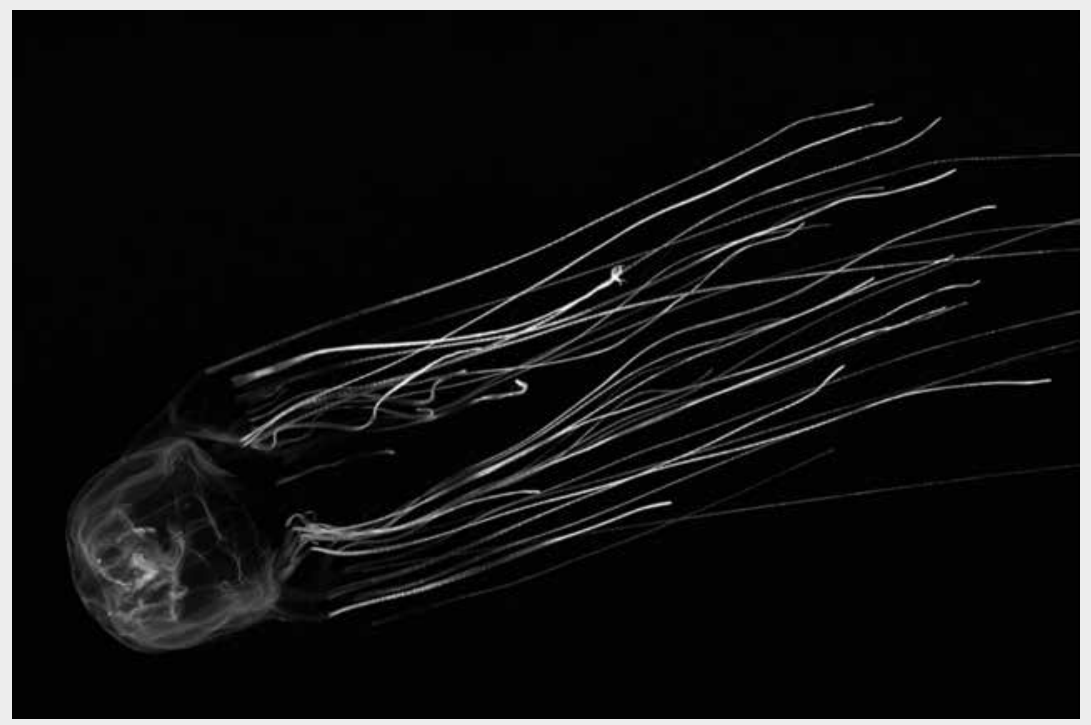

Fuente: http://photography.nationalgeographic.com/wallpaper/photography/photos/underwater-oddities/boxjellyfish-doubilet/

ellos se encuentra un médico ahogado en deudas, un pianista ciego, una mujer hispana abusada por su esposo y Emily. Cuando encuentra a Emily (interpretada por Rosario Dawson), una mujer con una grave insuficiencia cardíaca, se enamora de ella complicándose su proyecto. Ayudar a Emily implicará una decisión extrema.

Con las preguntas guías 1, 2 y 3 para el visionado del objeto de aprendizaje (Cuadro IV), se focaliza en el estudio de cuestiones disciplinares conceptuales de Zoología, referidas a la especie de Cnidario utilizada en el film. En esta parte del desarrollo de la propuesta se enseña la anatomía macro y micro de Chironex fleckeri utilizando figuras esquemáticas y fotografías que sirven como soporte visual. Cuadro V. Se establecen comparaciones entre la especie con otras medusas estudiadas en clases precedentes cuando se inició la unidad programática correspondiente al Phylum Cnidaria. Con base en las características anatómicas macroscópicas consideradas (forma, tamaño, disposición de los tentáculos, coloración) y otras de nivel micro (estructura y tipo de cnidocitos), se concluye en la ubicación taxonómica de la especie utilizada en el film: Clase Cubozoa. 
Con las preguntas guías 4 y 5 para el visionado del objeto de aprendizaje, se focaliza en cuestiones de Medicina referidas a los trasplantes de órganos. Por ejemplo, se analiza la utilización para trasplante de órganos provenientes de una persona muerta por un tóxico, donde se dio un shock y disfunción multiorgánica (fallo de todos los órganos). También se repara en el cambio de color en los ojos de Ezra luego del trasplante de córnea, y el reinicio -aparentemente espontáneo- del ritmo sinusal en Emily al culminar la operación.

La pregunta 6 orienta la observación y discusión a cuestiones jurídicas y legales. Se repara en la ausencia de maniobras de reanimación cardiopulmonar; también se denota la falta de investigación policial. Otro aspecto analizado es la posible configuración de delito por omisión y/o encubrimiento en aquellas personas que eran conocedoras de las intenciones de Tim.

Finalmente, con la pregunta guía del visionado número 7 se apunta a una valoración global del film. Se pone aquí en consideración algunas cuestiones éticas y morales, tales como la manipulación emocional y la postura de vida del protagonista. Compartiendo o disintiendo con las apreciaciones y valoraciones que hacen críticos de cine, los estudiantes expresaron su parecer en forma escrita. Aspiramos en este momento que la fundamentación del acuerdo o desacuerdo se vea enriquecido por el análisis que desde distintas disciplinas realizamos del epílogo del film.

\section{El cine como recurso didácti- co. Conclusiones de la propuesta implementada}

Compartimos con Zúñiga, Leiton y Naranjo (2014) que la enseñanza de las ciencias en la actualidad debe utilizar los medios de comunicación como herramienta de conocimiento que permiten vincular de manera muy favorable los avances científicos -tecnológicos con aspectos sociales.

Las producciones cinematográficas se presentan como un recurso privilegiado para la enseñanza de las ciencias bajo una mirada CTS. En la propuesta implementada el recurso sirvió para un abordaje interdisciplinar donde Zoología, Medicina y Derecho hicieron sus aportes para el análisis de los hechos que sostienen la trama argumentativa del film. Los contenidos específicos de la asignatura Zoo-I, los disciplinares conceptuales, fueron enseñados a partir de considerar la especie de Cnidario que se utiliza en el epílogo de la película.

La observación del relato cinematográfico y el análisis y discusión posterior, es una estrategia sumamente valiosa para aprender ciencia. Esto implica diseñar tareas de enseñanza y aprendizaje donde la pautación de consignas para observar el relato y analizarlo, es pieza clave. La orientación del visionado por una guía de actividades obliga a los estudiantes a modificar la actitud receptiva y pasiva que suele caracterizar a los espectadores, por una activa y crítica (Castro, 2006). La experiencia que hemos desarrollado imple- 


\section{Cuadro VI. Acuerdo de los estudiantes de Zoo-I en 2013/14, con valoraciones de la crítica especializada en cine}

Valoraciones

Valoración de crítica especializada*

I II III IV V VI

Porcentaje de estudiantes que acuerdan $\begin{array}{llllll}34 \% & 8 \% & 0 \% & 42 \% & 8 \% & 8 \%\end{array}$ con el crítico

* La numeración se corresponde con la que se presenta en el cuadro IV

Fuente: Elaboración propia

mentó estas características, y obtuvo resultados deseados.

Hemos logrado a través de la observación y análisis de una producción cinematográfica, movilizar conocimientos científicos, reflexionar con base en ellos y aplicarlos o usarlos en situaciones específicas. El uso de esta estrategia didáctica implicó la búsqueda de información, elaboración y reelaboración de lo conocido a fin de poder tender puentes entre la ciencia con lo que se presenta en la situación planteada en el film que se analiza.

Con esta estrategia didáctica propiciamos el desarrollo de espectadores críticos, atentos a los contenidos morales presentes en el film (Castro, 2006), así como también al sustento o apoyo científico de lo que se plantea en el relato. En palabras de Ferrés, se logró "establecer relaciones coherentes y críticas entre lo que aparece en la pantalla y la realidad del mundo por fuera de ella" (Ferrés, 1999).
Resumimos en el cuadro VI los resultados de la valoración global del film, en estudiantes que cursaron la asignatura Zoo-I en los años 2013 y 2014. En lo que respecta a contenidos morales del film analizado, vemos que los estudiantes mayoritariamente acuerdan con Todd McCarthy de la revista Variety $(42 \%$, ver Cuadro $\mathrm{n}^{\circ}$ VI). La mirada que hace este crítico resalta el cariz sentimental y de fábula que tiene el film. Un porcentaje también alto de estudiantes (34\%) acuerda con Roger Ebert del Chicago Sun-Times; él también señala el cariz manipulador del film por valerse de cuestiones sensibles de fuerte carga emocional.

Constatamos que el acuerdo de los estudiantes con la valoración de la crítica especializada, incorpora como argumento o fundamentación la escasa adecuación científica de lo que plantea el film. Las siguientes afirmaciones de estudiantes del curso de Zoo-I, sintetizan muy bien lo que afirmamos: 
Acuerdo con la crítica de Todd Mc Carthy. Al haber analizado la película en la clase sigo opinando que es una fábula sobre todo muy sentimental. Si se analiza racionalmente $y$ detenidamente, se pueden descubrir errores y fantasías... en la realidad no se podrían dar varios de los hechos que se presentan.

Como lo explica otra estudiante, los "errores" son explicados o fundamentados en las cuestiones médicas y legales que fueron analizadas en clase:

Desde el lente de la ciencia la película encierra errores... no se pueden donar órganos afectados por neurotóxicos así como tampoco hacer todo lo que hizo el protagonista de la película cuando existen en todos los paises normativas que regulan la donación de órganos.

Por otra parte las evaluaciones de los aprendizajes en contenidos específicos de la asignatura Zoo-I, realizados a través de las clásicas pruebas escritas semestrales, y luego también en escritos y orales de las instancias de exámenes, muestran un buen desempeño de los estudiantes en preguntas referidas al grupo Cubozoa. Todo indica que la significatividad y motivación que sobre estos animales se consiguió, redunda en buenos aprendizajes. Consideramos que la estrategia y recurso utilizado explican, en buena medida, el éxito educativo. Una estudiante del grupo que trabajó el tópico CTS n ${ }^{\circ} 1$, comentó:
A nuestro sub-grupo nos resultó muy interesante y motivante realizar la investigación bibliográfica para armar la monografía. Algunos compañeros habían visto la película pero no se habian cuestionado los hechos que se plantean en la trama argumentativa como lo hicimos en clase. Nunca se nos ocurrió que a partir de una película como esta se pudiera introducir el estudio de un grupo animal, en el liceo nunca hicimos algo parecido... fue una experiencia innovadora y creo que inadie en la clase se va a olvidar de la existencia y de las características de los Cnidarios Cubozoos!

Una vez finalizado el estudio del grupo Cubozoa, se consultó oralmente a los estudiantes de la generación 2014 sobre la estrategia utilizada para la enseñanza del tema. La valoración fue coincidente en un aspecto, que podemos resumir en los siguientes términos: experiencia innovadora que lleva a pensar la ciencia (Zoología y Medicina) como instrumento para entender una producción cultural de fuerte incidencia social e individual, el cine.

\section{Estrategias didácticas vividas en la Formación Docente inicial}

Más allá de la forma que adopte el currículo en la formación docente, más allá de las asignaturas y programas que lo conformen, es fundamental que el conocimiento disciplinar no se presente atomizado y fragmentado. Es por esto importante generar espacios 
y formas de trabajo que apunten a una visión integrada e interdisciplinar de las ciencias.

Las experiencias educativas vividas en la formación docente inicial tienen un fuerte impacto en la conformación del perfil profesional (Blanco, 1999; Vezub, 2002; Bolivar, 2007). Davini (1995) sostiene que los productos internalizados a lo largo de la historia escolar de los estudiantes -a lo cual nosotros agregamos a través de las estrategias didácticas aplicadas por los docentes- se constituyen en un "fondo de saber" que orientan en buena medida las formas de asumir su propio papel como docentes. Por esto es fundamental que en formación docente las prácticas educativas hagan eco en las máximas del saber popular: haz lo que se dice que se debe de hacer y, enseña con el ejemplo más que con el discurso.

El tratamiento de temas disciplinares bajo un enfoque CTS es una forma de integrar el conocimiento, promover la interacción e intercambio disciplinar y humanizar la ciencia. Es un enfoque que ayuda al estudiante a dar sentido y relevancia a lo aprendido. Es una forma de contribuir a que en la educación superior se de lo que López llama "cambios en los principios organizativos del conocimiento"; en este se busca pasar de estructuras curriculares agregadas, yuxtapuestas, enciclopédicas a estructuras curriculares integradas, interdisciplinarias, abiertas, y dialogantes. Lograr este cambio es un importante desafío para la educación superior (López, 2011).
La utilización del cine como recurso educativo en clases de ciencias experimentales es una opción didáctica que posibilita muy bien un enfoque CTS para temas programáticos de una asignatura. En buena parte del mundo actual las persona tienen acceso a las producciones del séptimo arte; internet con el visionado on-line sumado a la TV por cable, el alquiler de películas en DVD y la clásica TV por aire, hacen que el cine sea parte de la cotidianidad para la mayoría de las personas. La enseñanza de las ciencias debe contribuir al desarrollo de espectadores críticos, observadores y analizadores de la realidad que les toca vivir con bases sólidas. Desarrollar en los estudiantes la capacidad de distinguir en las producciones cinematográficas realidad de fantasía, realidad posible de imposible (o muy poco probable), es un objetivo para la educación actual y la enseñanza de las ciencias experimentales tiene importantes aportes para hacer.

\section{Referencias bibliográficas}

ACEVEDO DÍAZ, J. (2004). Reflexiones sobre las finalidades de la enseñanza de las ciencias: educación científica para la ciudadanía. Revista Eureka sobre Enseñanza y Divulgación de las Ciencias, 1 (1), 3-16.

BLANCO, N. (1999). Aprender a ser profesor/a. El papel del prácticum en la formación inicial. En F. ANGULO RASCO, J. BARQUÍN RUIZ y A. PÉREZ GÓMEZ (coords.), Desarrollo profesional del docente: política, 
investigación y práctica (379-398). Madrid: Akal.

BOLIVAR, A. (2007). La formación inicial del profesorado de secundaria y su identidad profesional. Estudios sobre Educación, 12, 13-30.

CASTRO SANTIAGO, M. (2006). El Cine como Instrumento de Socialización. Revista de Filosofía A Parte Rei, 47.

DAVINI, M. C. (1995). La formación docente en cuestión: política y pedagogía. Buenos Aires: Paidós.

FERRÉS, J. (1999). Televisión y educación. Barcelona: Paidós.

GONZÁLEZ, M., LÓPEZ, J. y LUJÁN, J. (1996). Ciencia, Tecnología y Sociedad: una introducción al estudio social de la ciencia y la tecnología. Madrid: Tecnos.

LÓPEZ, N. y PUENTES A. (2011). Modernización curricular de la Universidad Surcolombiana: integración e interdisciplinariedad. Revista Entornos, 24, 103-122.

LÓPEZ CEREZO, J. (1999). Los estudios de ciencia, tecnología y sociedad. Revista Iberoamericana de Educación. OEI - Ediciones, 20, 217-225.

LUGO BLANCO Á., HERNÁNDEZ ACOSTA R. y ÁLVAREZ YONG C.
(2012). ¿Cómo potenciar la educación ambiental, desde la disciplina Zoología, en la formación inicial de profesores de Biología-Química, de la UCP Rafael María de Mendive? Revista Órbita Científica, 18 (67), 2.

VEZUB, L. (2002). Los residentes en acción. Las tareas y preocupaciones en el proceso de inducción al magisterio. En M.C. DAVINI (coord.), De aprendices a maestros. Enseñar y aprender a enseñar (79-119). Buenos Aires: Papers Editores.

WILEY, D. A. (2000). Connecting learning objects to instructional design theory: a definition, a metaphor, and a taxonomy. En D. A. WILEY (ed.), The Instructional Use of Learning Objects. Recuperado de http://reusability.org/read/chapters/wiley.doc [24 de diciembre de 2014]

ZÚÑIGA MELÉNDEZ, A., LEITON, R. y NARANJO RODRÍGUEZ, J. (2014). Del sistema educativo tradicional hacia la formación por competencias: Una mirada a los procesos de enseñanza aprendizaje de las ciencias en la educación secundaria de Mendoza Argentina y San José de Costa Rica. Revista Eureka sobre Enseñanza y Divulgación de las Ciencias, 11 (2), 145-159. DOI: 10498/15972. 